\title{
Transform Residual K-means Trees for Scalable Clustering
}

\author{
Jiangbo Yuan \\ Department of Computer Science \\ Florida State University \\ Tallahassee, Florida 32306, \\ Email: jyuan@cs.fsu.edu
}

\author{
Xiuwen Liu \\ Department of Computer Science \\ Florida State University, \\ Tallahassee, Florida 32306 \\ Email: liux@cs.fsu.edu
}

\begin{abstract}
The $K$-means problem, i.e., to partition a dataset into $K$ clusters is a fundamental problem common to numerous data mining applications. As it is an NP-hard problem, iterative optimizations are typically used such as the $K$-means algorithm to compute cluster centers. As the $K$-means algorithm is exponential both in computation and storage in terms of required bits to encode cluster centers, approaches with low computation and storage complexity have been actively studied both for signal compression under real-time constraints and for clustering of large scale high-dimensional datasets. By generating cluster centers via Cartesian products of cluster centers in multiple groups or multiple stages, product and residual $K$-means trees reduce computation and storage complexity, making it possible to cluster large scale datasets. As residual $K$-means trees do not require assumed statistical independence among groups that are required by product $K$-means trees, they generally give better clustering quality. A known limitation of residual $K$-means trees is that the gain diminishes with added stages. In this paper, by identifying increased intrinsic dimensions of residual vectors with more stages as a limiting factor of performance due to the curse of dimensionality, we propose transform residual $K$-means trees as a generalization by applying cluster specific transforms to increase the correlations of residual vectors. Our methods substantially reduce the increase of intrinsic dimensions and therefore increase the effectiveness of multiple-stage residual $K$ means trees. Experimental and comparative results using widely used datasets show our methods give the best performance among all scalable methods. Furthermore, our methods enable effective super-clustering learning and provide scalable solutions to duplicate detection and other data mining problems.
\end{abstract}

\section{INTRODUCTION}

In many data mining applications, where large amounts of unlabeled data are available, a fundamental problem is to identify underlying patterns in the data so that additional steps can utilize such patterns for prediction and classification among many other usages. For example, practically an unlimited number of images are available on the Internet (from photo and video hosting sites and social media sites among others); these images are useful to build visual models that are effective under numerous conditions covered by the images; further applications such location inference can then infer locations of images (and videos), providing additional information to be used for social applications. More broadly, in this big data era, the amount of available data is growing exponentially [1] and it is computationally infeasible and costprohibitive to manually label the data. In these and many other scenarios, a commonly adopted processing framework is to first extract domain-dependent and application dependent features (such as SIFT features for images [2]); to be able to discriminate large number of classes effectively, such features need to be high dimensional. An essential next step is to identify groups, partitions, or clusters in the feature set, which leads to clustering. As a scheme of unsupervised learning, clustering plays a key role as a preprocessing step to group data sets.

Informally, the goal of clustering is to break data sets down to small and (hopefully) semantically meaningful groups/partitions w.r.t. some predefined pairwise distances and objective functions. As a fundamental tool that is used in many applications, clustering has been widely studied and many methods have been proposed beyond the K-means algorithm; see [3] for further information. However, such algorithms have been demonstrated to be ineffective for handling high dimensional large datasets even though they have been effective for problems that use low-dimensional smaller datasets. In attempts to overcome the limitations of traditional clustering algorithms and identify most favorable trade-offs among quality, computational and storage costs, and practical feasibility for large scale problems, there have been revived interests in clustering algorithms that are effective for large high dimensional datasets by imposing constraints on the tree structures to reduce the complexity for both computation and storage. Two families of methods, product quantization [4] and residual vector quantization [4], which were first studied for source coding as alternatives to achieve optimal performance under real-time constraints, have low complexity in both computation and storage, making scalable clustering feasible. Production quantization methods divide a high dimensional space into multiple subspaces, perform clustering in each subspace separately, and then use Cartesian product of the resulting cluster centers to generate an implicit tree structure. Quality degradation for such methods is due to the statistical dependencies among the subspaces that are not exploited and more restrictive quantization regions [4]. Residual vector quantization methods overcome these limitations by iteratively clustering residual vectors from the previous clustering stage. However, it has been observed that the effectiveness of residual vector quantization methods is limited to a very small number of stages due to increased randomness of residual vectors. In this paper, we propose to apply cluster-dependent transformations so that residual vectors of different clusters become more aligned, leading to increased performance even for a large number of stages. 
The rest of the paper is organized as follows. Section II gives a brief overview of different approaches toward scalable clustering of large datasets. In Section III we introduce local intrinsic dimensions and Section IV describes the proposed Transform Residual K-Means Tree method, to overcome increased intrinsic dimensions of residual vectors. In Section $\mathrm{V}$ we show systematic experimental results on large datasets to demonstrate the effectiveness of the proposed method. We conclude a paper with a summary and future work in Section VI.

\section{RELATED WORK}

The general problem of clustering can be defined mathematically as an optimization problem, commonly known as the " $K$-means" problem. The problem is to identify $K$ cluster centers such that they minimize the total scatter of all cluster centers. As it is an NP-hard problem [5], a commonly adopted solution is an iterative optimization algorithm, such as the $K$-means algorithm ${ }^{1}$. The $K$-means algorithm consists of two steps that are straightforward to implement: 1) in the classification step, given the current cluster centers, assign each sample to the nearest cluster center; 2) in the updating step, recompute each cluster center as the mean of all of the samples assigned to the cluster. The two steps are called iteratively until there is no change in cluster assignment.

The $K$-means algorithm can be directly applied to any dataset if there is no limit on computation, storage, and training samples and it provides a practical upper bound of achievable clustering performance in terms of the total scatter. When applied to large datasets in high dimension, both the computation and space complexity grow exponentially with the dimension [4]. In addition, for many data mining applications, often a large number of cluster centers is required in order to be able to provide useful, additional information. Traditional $K$-means algorithms can not provide a practical solution in such applications. Since the $K$-means algorithm provides an upper bound on the achievable solution, the research question naturally focuses on methods that can overcome the limitations due to $K$-means' complexity and at the same time provide performance as close as possible to the one achieved by the $K$ means algorithm or at least to be sufficient and useful for practical applications. In search for practically optimal solutions for signal quantization, the problems have been studied in the source coding community in order to obtain coding methods that give the best performance under real-time constraints [4]. Several families including tree-structured, partitioned ${ }^{2}$, and multi-stage methods ${ }^{3}$ have been proposed and some are being implemented and used in real-world systems.

Spatial partition trees (SP-trees) are very common data structures that are utilized to speed up clustering whenever computations is a bottleneck. Clustering-based tree structures, e.g. hierarchical $K$-means tree (HKM-tree), reduce the computational complexity from linear to logarithmic in terms of the number of cluster centers (from exponential to linear in terms of bits required to encode cluster centers). While such tree-structured methods reduce the computational complexity,

\footnotetext{
${ }^{1}$ The idea was first described in [6]; it is known as the generalized Lloyd algorithm in source coding community [4].

${ }^{2}$ Also commonly called production quantization [7]

${ }^{3}$ Commonly also called residual vector quantization [8].
}

the storage requirement of cluster centers remains exponential in terms of the dimension and the number of bits to encode the centers. Single projection-based trees are another spatial partition tree family, the storage for their structures are efficient by storing only one projection per node. Yet some conventional SP-trees, e.g. KD-trees, suffer from the curse of dimensionality. Recent theoretical work has shown that some advanced SP-trees, e.g. random projection tree (RPtree), principal direction tree (PD-tree/PCA-tree), and 2-Means tree (2M-tree), are adaptive to the intrinsic dimension of the data [9]. Among those methods, PCA-trees and 2-Means trees outperform KD-trees and RP-trees on clustering quality, see details in Section V. Yet they pay much more on construction costs. One reason is both PCA-trees and 2-Means trees select the splitting directions adaptive to the underlying data distribution, either by the principal direction or the connected clusters' centers direction. In fact, some recent active problems have employed those trees to improve efficiency. For instances, RPtrees were used for large-scale clustering problems [10] and $k \mathrm{NN}$-graph construction [11], and along with other trees they were evaluated under vector quantization and regression scenarios [9], approximate nearest neighbor search problems [12][15]. This points out a promising direction that by designing better SP-trees adaptive to intrinsic dimension to tackle both scalability issue and high-dimensional data.

Two structured clustering families of methods that reduce both the computation and storage requirements are partitioned methods and multi-stage methods. Partitioned methods divide the (high-dimensional) input vector into two or more subspaces and then apply traditional algorithms such as the $K$ means algorithm on each partitioned sub-space. Suppose that there are $M$ sub-vectors each with $k_{1}, k_{2}, \ldots, k_{M}$ cluster centers, the total possible number of cluster centers in the original space is $k_{1} \times k_{2} \ldots k_{M}$ given by the Cartesian product of the centers in the partitioned sub-vectors. Note that the partitioning is not limited to grouping of vector components, linear combinations of input vector can be used and other decompositions (such as the magnitude and direction representation) can be used as well [4]. Recently, this idea is directly applied to large scale data sets from computer vision and achieved substantial improvements in scalability [7].

While partitioned methods reduce the complexity both in computation and storage, performance degradation can be severe if there is substantial statistical dependence among the partitioned sub-vectors [4]; additionally, how to optimally partition the vector, while being studied [16], [17], is still an open problem. Multi-stage methods, while reducing both computation and storage complexity, also overcome the potential limitations of partitioned methods. The central idea is to apply clustering on the residual vectors resulting from the previous clustering stage. Similar to partitioned methods, the total possible number of cluster centers in the original space is large using the Cartesian product of the centers from all stages. A side effect of reducing the storage complexity is that it is possible to generate potentially very large number of clusters using multiple sub-vectors or multiple stages, which provides a solution to the scalability problem, one of the classic issues that challenges to conventional clustering methodologies.

However, there is no free lunch for multi-stage methods either. The gain of adding an additional stage relies on the ex- 
istence of commonality among residual vectors from different cluster centers. It has been observed that the shared structures diminish quickly as residual vectors become more random, limiting the effectiveness of multi-stage methods to two or three stages for signal compression [4] and in general a small number of stages, depending on the nature of the dataset.

In this paper, we propose a method to alleviate the problem of diminished commonality among residual vectors. The key idea is to transform the residual vectors for each cluster center so that the residual vectors from different clusters become and remain similar, in doing so to extend the effectiveness of multistage methods to more stages. Our systematic comparisons with other methods show improved performance; compared to the HKM-tree method, we have achieved at least one order of magnitude speed up with marginal loss in quality. Our method, named Transform Residual K-means Tree (TRKM tree) enables multi-stage methods to perform better than partitioned methods for multiple stages.

\section{LOCAL INTRINSIC DIMENSION}

The focus of this paper is to develop scalable solutions to clustering of large scale high dimensional datasets. Properties that only exhibit in high dimensional space such as distance concentration affects the effectiveness of the $K$-means algorithm. Since our methods build on multiple applications of the $K$-means algorithm, the so-called curse of dimensionality does not manifest directly in the proposed methods and we give general remarks in Section V. We introduce local intrinsic dimension, which was proposed to explain a key difference between a number of methods that suffer from the curse of the dimensionality and another group of methods that seem to be able to handle high dimensional data much more effectively.

There are a number of ways to define local intrinsic dimension. For data mining applications, a local covariance dimension is most relevant. According to Verma et al. [9], a set of points has covariance dimension $(d, \epsilon)$, where $0 \leq \epsilon \leq 1$, if the first $d$ dimensions with maximum variance capture at least $1-\epsilon$ fraction of the total variance in the set. In other words, if $d$ dimensions with maximum variance are used, one will loss at most $\epsilon$ fraction of the total variance in the set. Computing the covariance dimension of a set is straightforward by computing its covariance matrix and then sorting the eigenvalues of covariance matrix in descending order. The computation can be done on any set of points; when it is applied to points in a local neighborhood, it is called local intrinsic covariance dimension; when it is applied to an entire entire dataset, it is called global intrinsic covariance dimension.

Figure 1 shows the cumulative distribution of eigenvalues of different datasets, from which their intrinsic covariance dimension can be estimated. As shown in these examples, an intrinsic covariance dimension of a dataset provides a "measure" of intrinsic difficulty of the dataset, regardless of the dimensionality of the original data. A dataset with a higher intrinsic covariance dimension would be more difficult to model due to the curse of dimensionality than one with a lower intrinsic covariance dimension.

As shown in [9], methods that are sensitive to intrinsic covariance dimension are shown to be effective on real world large scale datasets while methods that are sensitive to the
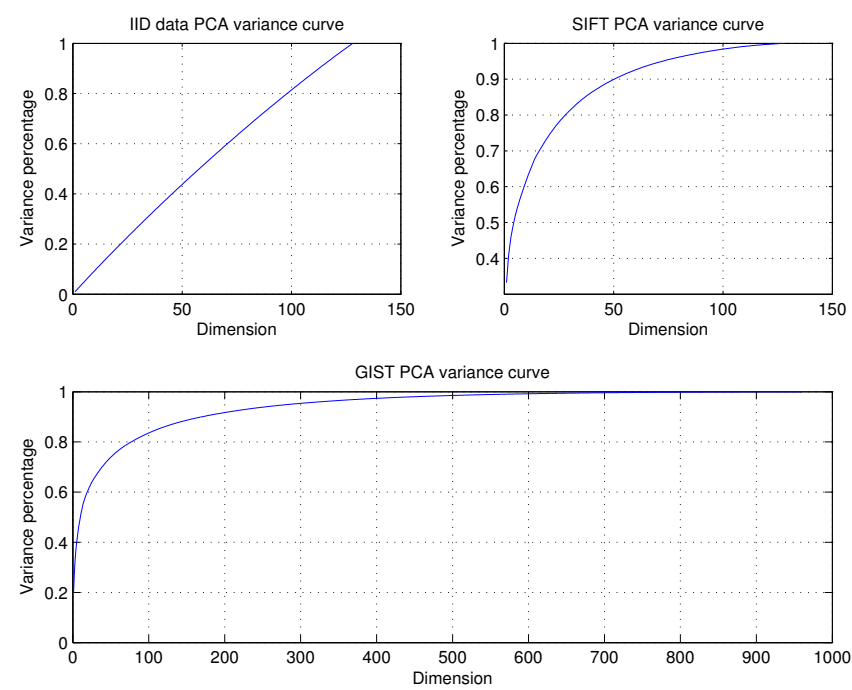

Fig. 1. PCA variance curve example for different data types.

dimension of the given data tend to suffer the curse of dimensionality when applied to the same datasets. In other words, if we can lower intrinsic covariance dimension of a dataset for a problem, more effective methods can be employed. We will use this property to identify problems with existing residual vector quantization methods and propose a solution to alleviate such problems.

\section{TRansform Residual $K$-Means TREeS}

\section{A. Residual K-Means Trees}

The central idea of residual $K$-means clustering is to apply recursively $K$-means clustering to the residual vectors from the previous stage, leading to a multiple stage clustering algorithm. So the entire dataset is being used in every stage. Suppose that the dataset at stage $i$ consists of $X_{1}^{i}, X_{2}^{i}, \ldots, X_{N}^{i}$ data points (as column vectors) with $i=1$ for the original data points. we obtain $K^{i}$ cluster centers,

$$
C^{i}=\left\{C_{1}^{i}, C_{2}^{i}, \ldots, C_{K^{i}}^{i}\right\},
$$

using the $K$-means algorithm on the dataset ${ }^{4}$. Given that $\tilde{X}_{j}^{i}$ is the closest cluster center to $X_{j}^{i}$,

$$
\widetilde{X}_{j}^{i}=Q^{i}\left(X_{j}^{i}\right)=\underset{c \in C^{i}}{\arg \min } d\left(X_{j}^{i}, c\right)
$$

its residual vector is given as

$$
X_{j}^{i+1}=X_{j}^{i}-\tilde{X}_{j}^{i},
$$

which will be input to the clustering of the next stage. Here $d(.,$.$) is the distance between two vectors and Euclidean$ distance is used in this paper. We also keep track of the index of the cluster center from equation 1 as $I\left(X_{j}^{i}\right)$, where $C_{I\left(X_{j}^{i}\right)}^{i}=Q^{i}\left(X_{j}^{i}\right)$, to be used later in this paper. By applying the $K$-means algorithm iteratively $m$ times on the resulting residual vectors, we have a sequence of cluster centers. Given an input vector $X=X^{1}$, to find its cluster centers in the resulting residual tree, we iteratively find the closest cluster

\footnotetext{
${ }^{4}$ Any other clustering algorithm can be used as well
} 
center $\widetilde{X}^{i}=Q^{i}\left(X^{i}\right)$ at stage $i$, compute its residual vector using equation (2), and repeat the steps for the next stage. The resulting cluster centers for $X$ are

$$
\left(\tilde{X}^{1}, \tilde{X}^{2}, \ldots, \tilde{X}^{m}\right) \text {. }
$$

The final reconstructed vector $\widetilde{X}$ for $X$ is

$$
\widetilde{X}=\sum_{i=1}^{m} \tilde{X}^{i}
$$

and the final error vector is simply the residual vector from stage $m, X^{m+1}$. The mean squared error (MSE) for a $m$-stage residual $K$-means tree, is

$$
M S E^{m}=1 / N \sum_{j=1}^{N}\left(X_{j}^{m}-\tilde{X}_{j}^{m}\right)^{T}\left(X_{j}^{m}-\tilde{X}_{j}^{m}\right),
$$

where ${ }^{T}$ indicates transpose operator. Adding a stage will reduce the MSE error in general and will not increase the MSE error in the worst case. By noting the MSE error without the last stage is simply

$$
M S E^{m-1}=1 / N \sum_{j=1}^{N}\left(X_{j}^{m}\right)^{T}\left(X_{j}^{m}\right) .
$$

As each cluster center is given as the mean of its closest samples, the cluster centers minimize $\mathrm{MSE}^{m}$. Since $\mathrm{MSE}^{m-1}$ is a special form of $\mathrm{MSE}^{m}$ with all the means to be zero, we have $M S E^{m-1} \geq M S E^{m}$ and in general

$$
M S E^{1} \geq M S E^{2} \cdots \geq M S E^{m} \text {. }
$$

Residual $K$-means trees provide a scalable solution to clustering of large datasets and a very large number of cluster centers can be generated through the Cartesian product of cluster centers at different stages. The performance of such methods can be further improved via a joint optimization of all the cluster centers [4], which will be furthered investigated.

While the MSE is guaranteed not to increase with added stages in residual $K$-means trees, it has been observed that the gain diminishes quickly; as a result, in practice, residual $K$-means trees are limited to mainly to two stages for signal compression [4], For the datasets used, residual $K$-means trees are effective for more stages, but the gain decreases with more stages; see Figs. 5 and 6 in the next section for quantitative examples. To identify causes of the problem, we have estimated the local intrinsic covariance dimension of residual vectors with respect to the number of stages. As shown in Fig. 2, local intrinsic dimensions increase with more stages. Note that stage zero is the intrinsic dimension of the original dataset and there is a significant increase after the first stage of the RKM.

By design, RKM forces the nodes at any level to be shared by all the paths to the level, which implicitly requires that residual vectors from different cluster centers should be similar, allowing common nodes to be used among all of them. However, as residual vectors from different clusters are added, they do not generally have similar distributions due to misalignment of axes, giving rise to increased local intrinsic covariance dimensions.
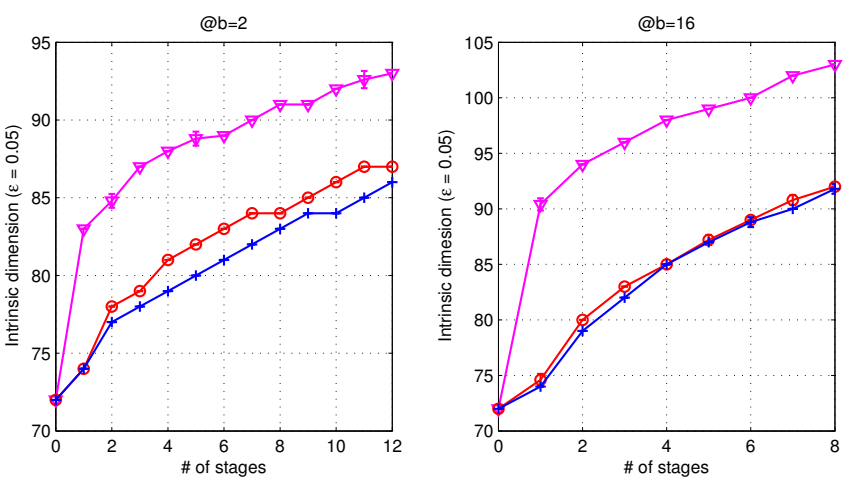

$\neg$ RKM - TRKM— — TRKM@FULL

Fig. 2. Intrinsic covariance dimensions (for $\epsilon=0.05$ ) of SIFT1M with respect to number of stages for the original RKM method, and proposed TRKM methods.

\section{B. Transform Residual K-means Trees}

To alleviate the misalignment of residual vectors from different clusters, in this paper we propose to apply a transform specific to each cluster so that the residual vectors are better aligned from different clusters. We associate each cluster center $C_{k}^{i}$ with a transform $T_{k}^{i}$, whose inverse $\left(T_{k}^{i}\right)^{-1}$ must exist. We then modify the residual vector given in equation (2) to

$$
X_{j}^{i+1}=T_{I\left(X_{j}^{i}\right)}^{i}\left(X_{j}^{i}-\tilde{X}_{j}^{i}\right),
$$

We call the family of generalized residual $K$-means trees Transform Residual $K$-means (TRKM) trees.

While our generalization only requires transforms to be invertible, the steps to show the non-increasing MSE error remain valid if transforms are orthogonal matrices, i.e., $\left(T_{k}^{i}\right)^{T} \times T_{k}^{i}=I$, (where $I$ is the identity matrix) because applying orthogonal matrices does not change the MSE error. However, in this case, scaling inhomogeneity among residual vectors from different clusters can not be accounted for by the transforms.

Computation wise, TRKM trees require an additional matrix-vector multiplication, the size of which is determined by the dimension of the input, which is small (see Section $\mathrm{V}$ for comparisons between TRKM trees and RKM trees). Note that the original RKM trees are a special case of TRKM trees with every $T_{k}^{i}$ to be the identity matrix.

The remaining question is how to estimate $T_{k}^{i}$, s that would be effective. There are several ways to achieve this. At any given stage, one way is to compute the optimal rotation to align every cluster to the center with the largest number of assigned data points, widely used in Procrustes shape analysis [18]. Another way is to align the axes of the residual vectors based on variance from different cluster centers, which can be implemented using principal component analysis of the residual vectors. For a very large dataset, accessing the entire dataset on disk can be slow and one-pass algorithm can be used. For $X_{1}, \ldots, X_{N}$, the covariance matrix can be written as

$$
\frac{\sum_{i=1}^{N} X_{i} \times X_{i}^{T}-\left(\sum_{i=1}^{N} X_{i}\right)\left(\sum_{i=1}^{N} X_{i}\right)^{T} / N}{N} .
$$


By keeping the two summations, one-pass algorithm can be implemented in a straightforward way.

In this paper, due to space limitations, we use $T_{k}^{i}$, s computed using one-pass PCA; additionally, we also use variations where transforms are applied only to the first stage. Procrustes shape analysis methods are being exploited and comparisons of these two methods will be reported in other studies.

Figure 2 shows the effectiveness of transforms on limiting the increase of intrinsic dimensions with more stages. Clearly the transforms reduce substantially intrinsic dimensions; even transforms applied at the first stage only help reduce intrinsic dimensions. Note that effects of intrinsic dimensions are exponential in nature and the reduction achieved by the transforms is significant and their effects on performance are shown in the next section.

\section{EXPERIMENTAL RESULTS AND COMPARATIVE ANALYSIS}

In this section, we present our experimental results along with comparative analysis to other methods and parameter and implementation variations of transform residual $K$-means trees. There are two parameters required to be tuned for all examined methods relying on $K$-means, e.g. PKM (Productquantization K-Means), RKM (Residual-vector K-Means), and TRKM (see Section V.D for more). The $b$ value is the number of cluster centers on each sub-spaces/stages; and the $m$ is the total number of sub-spaces/stages. Then the amount of reachable cells or leaves on a tree equals to $b^{m}$. When $b=256$ and $m=8$, for instance, the total number of virtual cells/centers is $256^{8}=2^{64}$. However, the stored centers are only $b=256$ vectors for PKM or $b * m=2048$ vectors for RKM and TRKM. The product codebooks are often used to high-resolution vector quantization.

There are two configurations for our methods to be included, which are called TRKM and TRKM@FULL. TRKM applies the transforms computed using PCA only at the first stage while TRKM@FULL computes and applies the transforms in all the stages. Note that transforms can be selectively applied to any combinations of stages and TRKM@FULL provides an upper bound of our methods in terms of performance and a lower bound in terms of computational efficiency.

All the results have been produced using a computer with a single $2.3 \mathrm{GHz}$ AMD processor (with $512 \mathrm{~K}$ cache size), and 64 GB RAM. All methods have been implemented in MATLAB except the $\mathrm{C}$ library $\mathrm{Yael}^{5}$, of which the $K$-means function and a nearest neighbor search function have been used.

\section{A. Datasets Used}

The SIFT [2] and GIST [19] descriptor datasets we used were introduced in [7] and [20]. Table I shows general statistics of these datasets. SIFT50M and SIFT100M consist of the first 50 and 100 millions, respectively, vectors from the ANNSIFT1B set ${ }^{6}$. The size of training sets in Table $I$ is only for the $K$-means learning if not explicitly stated otherwise; the training sets are generated by sampling randomly from the corresponding full datasets.

\footnotetext{
${ }^{5}$ Available from https://gforge.inria.fr/projects/yael/.

${ }^{6}$ Obtained from http://corpus-texmex.irisa.fr/.
}

TABLE I. StATISTICS OF DATASETS USED.

\begin{tabular}{lccc}
\hline Dataset & dimension & \# all samples & \# training samples \\
\hline SIFT1M & 128 & $1,000,000$ & 100,000 \\
SIFT50M & 128 & $50,000,000$ & 100,000 \\
SIFT100M & 128 & $100,000,000$ & 1000,000 \\
GIST1M & 960 & $1,000,000$ & 100,000 \\
\hline
\end{tabular}

\section{B. The Distortion Measurement}

As we restrict our clustering problems only on Euclidean distance. Then it is inherently consistent to a vector quantization problem. In this case, the mean squared error (MSE) that is used to evaluate quantizers can be directly fit to our goal. Equation 3 is formulated specially for RKM. Here we re-write the MSE equation using more general terms.

$$
M S E=1 / N \sum_{j=1}^{K} \sum_{X_{i} \in G_{j}}\left\|X_{i}-C_{j}\right\|_{2}^{2},
$$

where $G_{j}$ consists of all the data points in the entire dataset assigned to cluster $j$ and $C_{j}$ is the cluster center. We will compare our method to RKM and PKM as well a few projection-based partitioning trees. The projection-based trees do not generate codebooks. For the sake of comparison, we will calculate the means of all leaf cluster centers. Whenever this mean re-calculation is taken, we will call the result MSE as global distortion, and otherwise reconstruction error following up a tree path is used as local distortion.

\section{The Scalability of TRKM}

Compared to conventional tree partitioning, e.g. hierarchical $K$-means trees (HKM-trees), our method enables to construct a tree only relying on a small number of training samples. This advantage is a side effect of the samples sharing at each stage. For clustering with small numbers of centers, e.g., $\leq 256$, the $K$-means algorithm is not so sensitive to the size of training set. This observation makes RKM/TRKM a possibility to tackle large-scale clustering problems by using relatively small training sets. By avoiding expensive learning process using a small training set, the whole clustering complexity on large datasets will be reduced accordingly. Figures 3 and 4 show TRKM with variant sizes of training samples. The gain is significant in computational complexity with almost no loss in the clustering quality.

As discussed earlier, we can simply use one-pass PCA on any dataset. Yet it is possible to further reduce the computational cost by training PCA on small training sets as we did for $K$-means. Our experimental results on a large dataset SIFT100M have given a strong support to the above idea. We observed that the one-stage TRKM can reduce $10 \%$ local distortion over PKM@ natural and 5\% to RKM, with parameters of $b=256, m=8$, where we only used 1 million training samples for both the $K$-means and PCA learning. The total computational overhead was limited to a few minutes that is negligible small compared to 150 minutes of the total cost of RKM. In the following experiments we also use the one-pass PCA learning on the full data set to examine the full strength of the proposed methods by using all samples for the PCA 

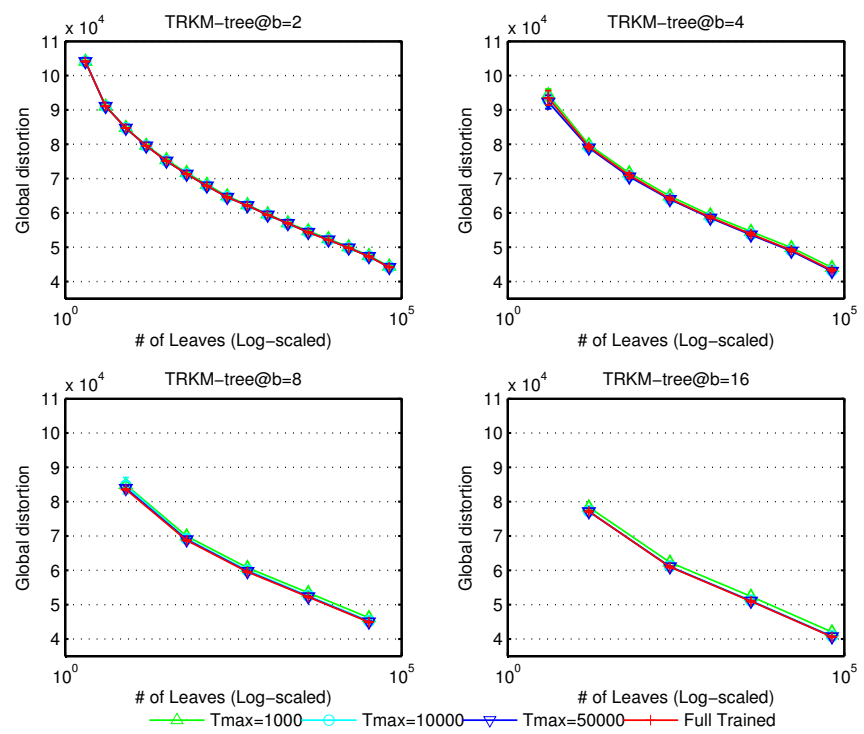

Fig. 3. The quality performance of our method with different sizes of training sets on SIFT1M dataset.
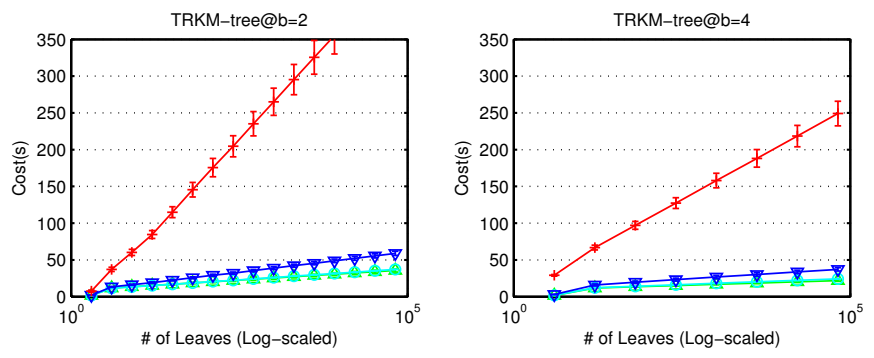

TRKM-tree@b=8
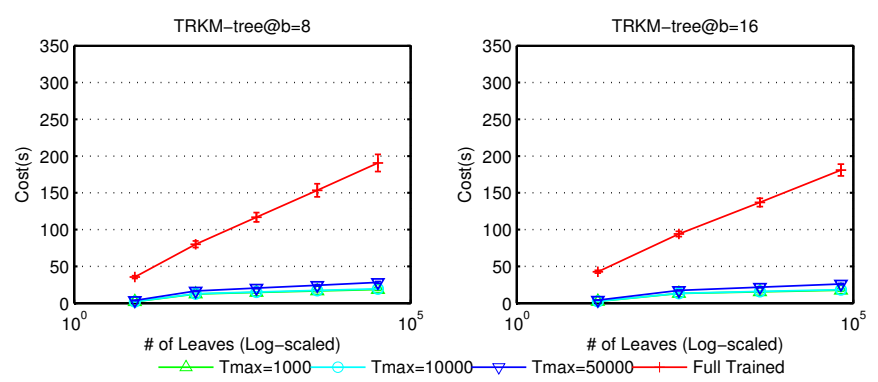

Fig. 4. The speed performance of our method with different sizes of training sets on SIFT1M dataset.

learning. In addition, we demonstrate that the PCA learning is not a main constraint in terms of computational cost.

\section{Clustering Performance of TRKM}

The scalability issue of clustering is not only of the size of samples $N$, but also the required number of cluster centers $K$. There are many problems that require extremely fine codebooks, where $K>N$ or $K>>N$. Near-duplicate detection problems in multi-media context [21], and data compression for resource efficient algorithms [7], [20] are typical examples. Here we classify such problems as a category of super-clustering. As the lower cost of both codebook storage
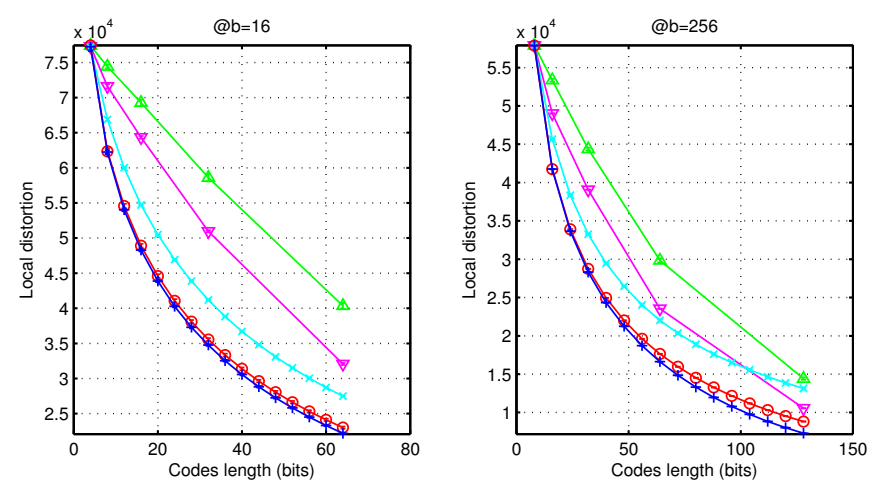

$\triangle$ PKM@random— $\nabla$ PKM@natural —x-RKM - - TRKM — — TRKM@FULL

Fig. 5. Super-clustering performance on SIFT1M dataset.
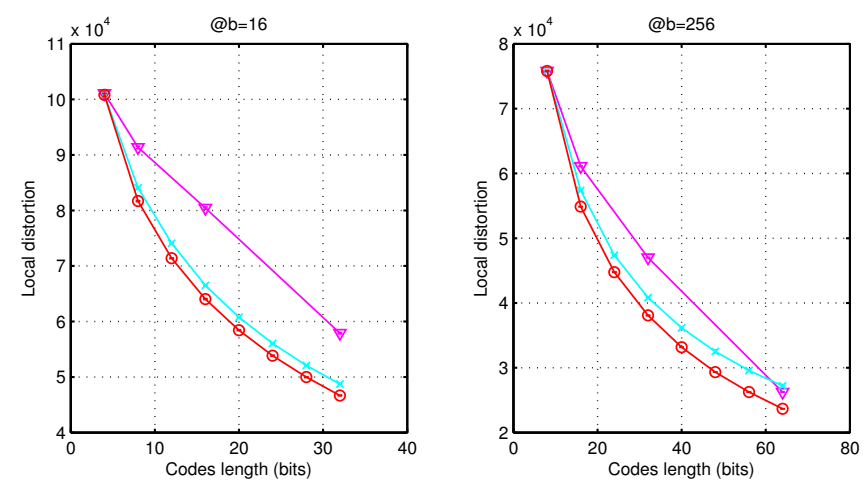

$\longrightarrow$ PKM@natural — $\times$ RKM -0 TRKM

Fig. 6. Super-clustering performance on SIFT50M dataset.

and computational cost of product vector quantization (PQ) and residual/multi-stages vector quantization (RVQ/MSVQ). They are the only known clustering-based techniques that can be applied to super-clustering ${ }^{7}$ and both of $K$-means versions of PQ and RVQ/MSVQ have been applied to different applications [7], [8], [21]. Here we call them by PKM and RKM respectively.

Figures 5 and 6 show the results for both PRK, RKM, and the proposed methods. Since super-clustering problems require much larger number of clusters related to the source coding, here we use code length $(L)$ to implicitly represent the clustering scales. If we have $m$ stages/sub-spaces, then $L=m \times \log (b)$ and the total cluster number $K=2^{L}$. The total storage for the compressed database is $L \times N$ bits. Here we present typical values of $b=16$ and $b=256$ in our results. Smaller numbers of centers, e.g. $b=16$, are useful for applications such as near-duplicate detection while $b=256$ is widely used for data compression since it implicates of 8-bits (1-byte) codes that are efficient for storage.

As pointed out earlier, the effectiveness of PKM depends

\footnotetext{
${ }^{7}$ Another option may consider the family of locality sensitive hashing (LSH) that are random projection based methods [22], [23], which in some sense is a special case of scalar quantizers.
} 
on statistical dependence among sub groups. As shown in Fig 5, there are substantial differences between PKM@ random and PKM@natural. Here PKM@random means that we randomize the coordinates before applying PKM; for the SIFT dataset, PKM@natural follows the natural groups of the SIFT, and generates groups that are statistically more independent. In general, finding effective groups may not be straightforward.

One key advantage of RKM and TRKM over PKM is that they do not decompose input vectors into smaller groups and therefore they do not require the statistical independence assumption among different groups. As shown in Figs. 5 and 6 RKM and TRKM give overall superior performance to PKM. However, the gain of RKM decreases significantly on later stages due to the diminished commonality among residual vectors from different clusters. By addressed this issue using PCA alignment transforms, TRKM methods produce the lowest local distortion in all cases. The improvement achieved over PKM is significant, where $30 \%-50 \%$ distortions are reduced for most configurations. At the same time, our methods give roughly $10 \%-20 \%$ improvement over RKM on SIFT1M and 4\% - 15\% improvement on SIFT50M.

It is not surprising that transforms in the first stage provide most improvement compared to subsequent transforms, due to that transforms in the second stage and beyond are not directly applied to real clusters but virtually merged clusters. The transforms from the first stage seem to align the merged clusters for subsequent stages, leading to reduced intrinsic dimensions and improved performance already.

The GIST feature descriptor is also a structured vector similar to SIFT but applied to an entire image. Compared to SIFT datasets, transform computation on 960-dimension GIST is more expensive. As shown in Fig. 7, our methods also provide a good trade-off between quality and complexity with very limited loss of quality but with substantial gain in complexity reduction.

Figure 7 also shows numerical results with respect to both clustering quality and speed for projection based partition trees. We also introduce an improved version of KD-trees named KD-tree:+10. It is different from conventional KD-trees by dynamically selecting splitting axis as the one with greatest variance from the first $l+10$ axes. Where $l$ is the ID of current tree level and all the axes have been presorted once at the root level. We use this improved version of KD-trees since it improves quantization quality while with very limited speeding overhead. While a RP-tree selects splitting axis as an random direction on each level, it is actually a special case of KD-trees.

Here we use only binary trees and global distortion for all trees as these trees are optimized for binary trees. As shown in Fig. 7, PCA-trees and HKM-trees overall achieved the best quality. However, they were also most expensive in terms of construction cost, mainly due to the learning over either PCA or $K$-means. Here, we have restricted TRKM and RKM to use only $10 \mathrm{~K}$ random samples for the $K$-means so both of them achieve much better quality while with comparable cost to KD-trees and RP-trees, especially on SIFT dataset. Even we limit PCA-based transforms in the first stage for TRKM, it still improves substantially relative to RKM in terms of global distortion.

One of the by-products of using $K$-means is that the $K$ -
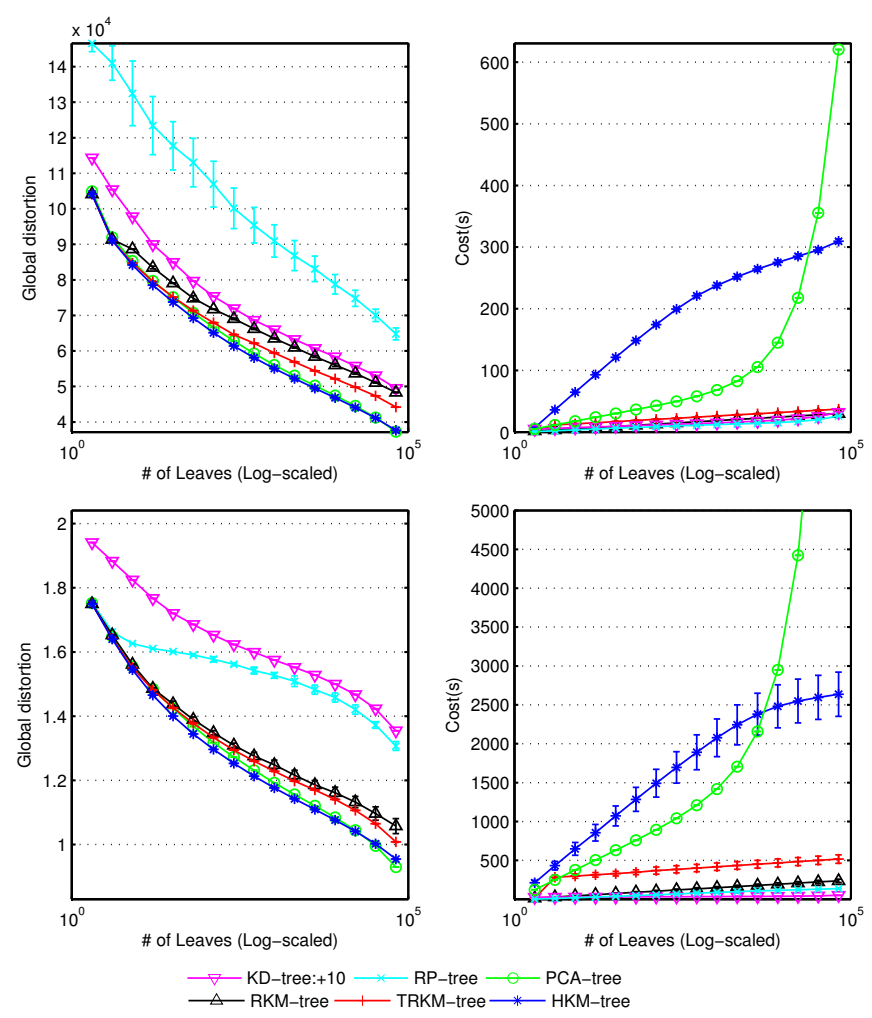

Fig. 7. Vector quantization performance of different trees on SIFT1M (top) and GIST1M (bottom) datasets.

means based approaches have more flexibility on the choice of branching factor $-b$. By fixing the ultimate output partitions, or the number of leaves on the tree, increasing $b$ will reduce the tree height. This flexibility is significant because in a search tree, for example, it will reduce the required parameters for a query. Figure 8 shows clearly the increased values of $b$ degrade the performance of all projection based trees. On the contrary, the qualities were slightly improved overall for all $k$-means based trees along with increasing the branches. The lower trees are especially favorable for nearest neighbor search trees as observed in [8], [14], [24];

\section{CONCLUSION AND FUtURE WORK}

In this paper we have proposed a new family of multistage methods, called Transform Residual $K$-Means Trees, for clustering. Our methods, by reducing the complexity both in both computation and storage, enable multi-stage methods to be effective for stages beyond a limited small number. We demonstrate via systematic experimental results the advantages of a particular realization of our methods (where transforms are computed using PCA of residual vectors) compared to other approaches. On the datasets we have used, we have achieved at least one order of magnitude improvement in speed with very limited loss of quality compared to the results from the HKM-trees algorithm, which is possibly an upper bound of performance for all constrained methods, including ours.

In this paper the transforms are computed using PCA of residual vectors; we are also implementing Procrustes shape 

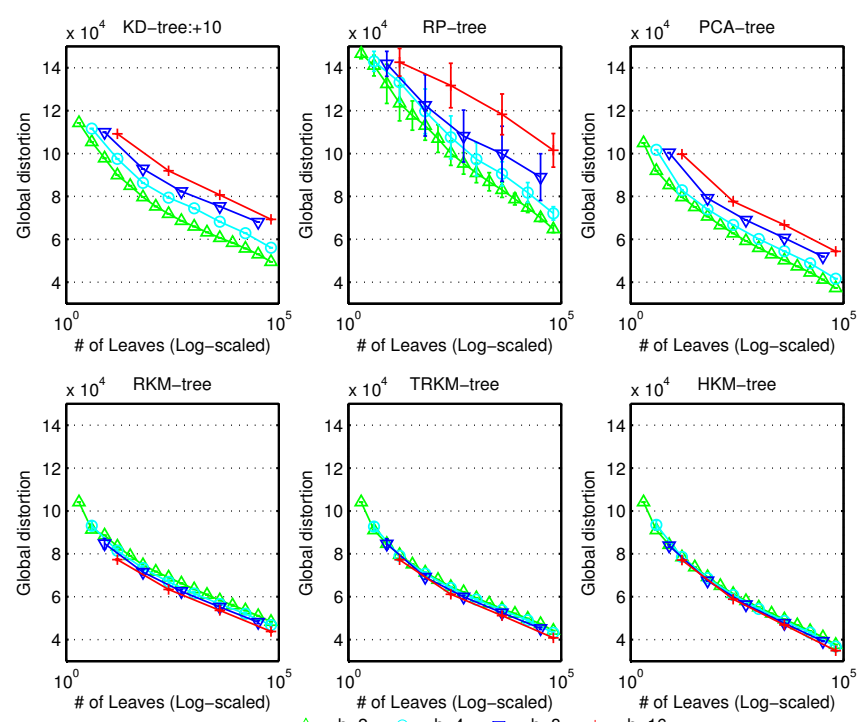

Fig. 8. Using multi-branch to examine flexibility of different trees, on SIFT1M dataset.

analysis methods and comparing the two methods. As our methods allow any invertible transform to be used, a subsequent research question is whether there are optimal transforms for a given dataset and how to compute such transforms. While allowing invertible transforms can handle scaling differences in residual vectors in different clusters, there is no longer theoretical guarantee that mean squared error will not increase with added stages. Joint optimization of transforms and all cluster centers may render this issue irrelevant. Clearly optimal transforms need to be investigated further.

Our methods can be used in a number of additional applications including efficient approximate nearest neighbor search, near duplicate detection, multi-media retrieval, data compression, high frequency financial data modeling, and in general data-driven intelligence. These problems are being investigated.

While our methods are proposed for high dimensional data, the curse of dimensionality does not manifest directly. However, the curse of dimensionality makes difficulty for the $K$-means algorithm in finding a solution close to the optimal solution in addition to the intrinsic sparsity issue. In TRKM, such effects may be limited due to relative small number of cluster centers in each stage. In addition, our methods use an approximation to the flat cluster centers to reduce complexity and the curse of dimensionality may also limit the effectiveness of the approximation. Quantifying these effects is being studied.

\section{REFERENCES}

[1] X. Wu, V. Kumar, J. Ross Quinlan, J. Ghosh, Q. Yang, H. Motoda, G. J. McLachlan, A. Ng, B. Liu, P. S. Yu, Z.-H. Zhou, M. Steinbach, D. J.
Hand, and D. Steinberg, "Top 10 algorithms in data mining," Knowl. Inf. Syst., vol. 14, no. 1, pp. 1-37, Dec. 2007.

[2] D. G. Lowe, "Distinctive image features from scale-invariant keypoints," IJCV, vol. 60, pp. 91-110, November 2004.

[3] A. K. Jain, M. N. Murty, and P. J. Flynn, "Data clustering: a review," ACM Comput. Surv., vol. 31, no. 3, pp. 264-323, Sep. 1999.

[4] A. Gersho and R. M. Gray, Vector quantization and signal compression. Norwell, MA, USA: Kluwer Academic Publishers, 1991.

[5] M. Mahajan, P. Nimbhorkar, and K. Varadarajan, "The planar kmeans problem is np-hard," in Proceedings of the 3rd International Workshop on Algorithms and Computation, ser. WALCOM '09. Berlin, Heidelberg: Springer-Verlag, 2009, pp. 274-285.

[6] E. Forgey, "Cluster analysis of multivariate data: Efficiency versus interpretability of classification," Biometrics, vol. 21, no. 3, pp. 768769, 1965.

[7] H. Jégou, M. Douze, and C. Schmid, "Product quantization for nearest neighbor search," PAMI, vol. 33, no. 1, pp. 117-128, Jan 2011.

[8] Y. Chen, T. Guan, and C. Wang, "Approximate nearest neighbor search by residual vector quantization," Sensors, vol. 10, no. 12, pp. 11259 $11273,2010$.

[9] N. Verma, S. Kpotufe, and S. Dasgupta, "Which spatial partition trees are adaptive to intrinsic dimension?" in Proceedings of the TwentyFifth Conference on Uncertainty in Artificial Intelligence, ser. UAI '09. Arlington, Virginia, United States: AUAI Press, 2009, pp. 565-574.

[10] J. Wang, J. Wang, Q. Ke, G. Zeng, and S. Li, "Fast approximate kmeans via cluster closures," in CVPR, 2012.

[11] J. Wang, J. Wang, G. Zeng, Z. Tu, and S. Li, "Scalable $k$-nn graph construction for visual descriptors," in In CVPR, 2012.

[12] J. McNames, "A fast nearest-neighbor algorithm based on a principal axis search tree," IEEE Trans. Pattern Anal. Mach. Intell., vol. 23, no. 9, pp. 964-976, Sep. 2001.

[13] C. Silpa-Anan and R. Hartley, "Optimised kd-trees for fast image descriptor matching," in CVPR, 2008, pp. 1-8.

[14] M. Muja and D. G. Lowe, "Fast approximate nearest neighbors with automatic algorithm configuration," in ICCVTA, VISSAPP'09, 2009, pp. 331-340.

[15] H. Lejsek, B. T. Jónsson, and L. Amsaleg, "Nv-tree: nearest neighbors at the billion scale," in Proceedings of the 1st ACM International Conference on Multimedia Retrieval, ser. ICMR '11. New York, NY, USA: ACM, 2011, pp. 54:1-54:8.

[16] T. Ge, K. He, Q. Ke, and J. Sun, "Optimized product quantization for approximate nearest neighbor search," In CVPR, 2013.

[17] M. Norouzi and D. Fleet, "Cartesian k-means," In CVPR, 2013.

[18] I. Dryden and K. Mardia, Statistical shape analysis, ser. Wiley series in probability and statistics. Chichester [u.a.]: Wiley, 1998.

[19] A. Oliva and A. B. Torralba, "Modeling the shape of the scene: A holistic representation of the spatial envelope," IJCV, vol. 42, pp. 145$175,2001$.

[20] H. Jégou, R. Tavenard, M. Douze, and L. Amsaleg, "Searching in one billion vectors: re-rank with source coding," in ICASSP, Prague Czech Republic, May 2011, QUAERO.

[21] J. Yuan, G. Gravier, S. Campion, X. Liu, and H. Jégou, "Efficient mining of repetitions in large-scale tv streams with product quantization hashing," in ECCV Workshops (1), 2012, pp. 271-280.

[22] P. Indyk and R. Motwani, Approximate nearest neighbors: towards removing the curse of dimensionality. ACM, 1998, vol. pages, pp. 604-613. [Online]. Available: http://portal.acm.org/citation.cfm?id= $276876 \& d l=$

[23] M. Datar and P. Indyk, "Locality-sensitive hashing scheme based on p-stable distributions," in SCG'04. ACM Press, 2004, pp. 253-262.

[24] J. Yuan and X. Liu, "A novel index structure for large scale image descriptor search," in ICIP, 2012, pp. 1937-1940. 